\title{
Hippocampal Tissue Transplants Reverse Lesion-Induced Spatial Memory Deficits in Zebra Finches (Taeniopygia guttata)
}

\author{
Sanjay N. Patel, ${ }^{1}$ Nicky S. Clayton, ${ }^{1,2}$ and John R. Krebs ${ }^{1}$ \\ ${ }^{1}$ Departments of Zoology and Pharmacology, University of Oxford, Oxford OX1 3PS, United Kingdom, and 'Section of \\ Neurobiology, Physiology, and Behavior, Division of Biological Sciences, University of California at Davis, \\ Davis, California 95616
}

The avian hippocampal formation (Hf) plays an important role in spatial memory for food storing. Here we examined the effects of excitotoxic lesions of the $\mathrm{Hf}$ and subsequent neural transplantation on a one-trial associative memory task in zebra finches. The results showed (1) that small ibotenic acid lesions of the dorsal Hf of zebra finches produced significant spatial memory impairments compared with controls, sham-lesioned birds, and prelesion performance; and (2) that Hf-lesioned birds given transplants of embryonic hippocampal $(\mathrm{H})$ tissue, but not those given transplants of embryonic anterior telencephalon (AT) tissue, showed a significant reversal of the performance deficits on the spatial memory task. Lesioned-only birds and lesioned birds given $\mathrm{H}$ or AT transplants that did not survive did not show behavioral improvement. Sham-lesioned and untreated control birds maintained good performance throughout the experiment. The $\mathrm{H}$ and AT transplants were found to be growing partially within the $\mathrm{Hf}$ and partially within the underlying ventricle. The transplants appeared healthy and contained neurons with beaded and unbeaded fibers (shown by immunohistochemistry with antibodies to parvalbumin, substance $\mathrm{P}$, and a $200 \mathrm{kDa}$ neurofilament protein). Blood vessels and erythrocytes were also present within the transplants. The results show that neural transplants can survive within the bird brain and that small lesions of the Hf produce significant spatial memory deficits that can only be reversed by surviving homologous $\mathrm{H}$ transplants, and not by heterologous telencephalon transplants.

Key words: learning and memory; neural transplantation; hippocampus; telencephalon; ibotenic acid lesion; birds; zebra finch
Neural tissue transplantation strategies have been widely used to study the principles of brain development and to ascertain structure-function relationships of particular brain regions (for reviews, see Gage and Fisher, 1991; Sinden et al., 1995; Stein and Glasier, 1995). The cognitive functions of the mammalian hippocampal formation (mHf), particularly learning and memory, have been studied successfully through the application of lesioning and neural transplantation techniques. Lesions of the mHf can disrupt performance on many different behavioral tasks (O'Keefe and Nadel, 1978; Jarrard, 1993; Eichenbaum, 1996), some of which can be ameliorated through the implantation of neural tissue into the hippocampus. For example, cerebral ischemia produces a lesion of the CA1 cell field of the hippocampus, with corresponding learning and memory deficits in the water and radial maze tasks (Auer et al., 1989; Davis and Volpe, 1991). These deficits can be reversed by the transplantation of either fetal hippocampal $(\mathrm{H})$ tissue or fetal CA1 cells into the lesioned hippocampus, but not by the grafting of other cell types (Onifer and Low, 1990; Netto et al., 1993). Thus, only homologous and not heterologous neural tissue transplants appear to be successful in ameliorating cognitive deficits produced in these Hf lesion models.

Received Sept. 30, 1996; revised Jan. 28, 1997; accepted Feb. 21, 1997.

This work was supported by a Biotechnology and Biological Sciences Research Council (BBSRC) postdoctoral research assistanceship to S.N.P. and grants from the BBSRC to N.S.C. and J.R.K. and by the Royal Society (J.R.K.). We wish to thank Alan Woodington and David Wilson for care of the birds. We are grateful to Prof. David Smith for the use of the facilities of the Pharmacology Department.

Correspondence should be addressed to Prof. J.R. Krebs, Department of Zoology, University of Oxford, Oxford OX1 3PS, UK.

Copyright (C) 1997 Society for Neuroscience $0270-6474 / 97 / 173861-09 \$ 05.00 / 0$
The avian hippocampal formation (Hf) is believed to be homologous to the mHf (for review, see Bingman, 1993) and is also involved in learning and memory. Food-storing birds use spatial memory for the accurate retrieval of food stored in numerous storage sites in the environment and have a larger Hf, containing more neurons relative to the rest of the telencephalon, than do nonstoring species (Healy and Krebs, 1992). Experience of food storing and retrieval also results in $\mathrm{H}$ enlargement with an increase in neuronal number (Clayton and Krebs, 1994). Bilateral aspiration lesions of the avian HF disrupt spatial discrimination learning and a food-storing bird's memory for the locations of food caches but does not affect caching and feeding behavior (Sherry and Vaccarino, 1989). Hf damage also affects a pigeon's ability to navigate home successfully from novel release sites (Bingman, 1990).

The experiment reported here was designed to study the effects of excitotoxic lesions of the hippocampus of zebra finches (Taeniopygia guttata) with subsequent neural transplantation of fetal homologous $\mathrm{H}$ or heterologous telencephalon tissue. A one-trial associative learning task with spatial (place) and nonspatial (color) components was devised that tested the bird's learning and memory abilities within its home cage. It was hypothesized that lesioned birds would show a performance detriment in the associative memory task and that this behavioral impairment would be reversed only by $\mathrm{H}$, but not by telencephalon, transplants.

\section{MATERIALS AND METHODS}

The subjects were 22 male and 25 female sexually mature, captive-bred adult zebra finches (Taeniopygia guttata). Zebra finches were also time mated for obtaining embryos at specific ages. Each bird was housed in single-sex groups of up to six in double cages measuring $92 \times 38 \times 31 \mathrm{~cm}$ 
$(\mathrm{W} \times \mathrm{H} \times \mathrm{D})$. They were maintained on a 10:14 light/dark cycle at a temperature of $21^{\circ} \mathrm{C}$. The lights came on at 8 A.M. and went off at 6 P.M. Food and water were provided daily ad libitum .

The one-trial associative memory task. Birds were trained and tested individually in the home cage. The cages could be separated in half by placing a wooden (opaque) divider vertically in the center of the cage. This ensured that each bird was trained and tested individually in half of the cage, while the other birds remained in the other half. The task consisted of two phases separated by a retention interval of $30 \mathrm{~min}$. In phase I, an experimental tray $(10 \times 20 \times 1 \mathrm{~cm})$ with 50 equal-sized compartments was introduced into the test side of the cage. Only one compartment contained $5 \mathrm{ml}$ of bird seed (which filled the compartment), and this compartment was covered with a visually distinct cardboard flap (with a unique color and pattern). Six other cardboard flaps covered six different empty compartments, chosen randomly. Each bird was given 5 min to find the food by removing as many flaps as necessary and then allowed to eat for $30 \mathrm{sec}$ so that it consumed some, but not all, of the seed. The tray was removed during the retention interval, and the bird rejoined the group. At the start of phase II 30 min later, the tray was reintroduced into the home cage. The bird obtained a reward in phase II by returning and removing the flap covering the compartment where it had eaten part of the food $30 \mathrm{~min}$ earlier in phase I. The test ended when the bird had eaten all the remaining food or after $5 \mathrm{~min}$, whichever occurred sooner. The number of looks the bird made to find the food, i.e., the number of flaps the bird removed in phases I and II, was recorded. Revisits to the same compartment were not counted as looks, because the food was visible once the flap had been removed. Each trial was unique in terms of both the individual flaps used and the spatial location of the baited and unbaited flaps. In phase I, performance was random, because the food was hidden, and therefore, birds should have lifted four flaps on average to find the food (because seven compartments were covered). Performance in phase II tests for memory in relocating the partially eaten food; if the birds had used memory to solve the task in phase II, then they should have lifted significantly fewer than four flaps, and a score of one look would have indicated perfect performance.

There were two types of memory tests-space and color-which differed in the cues available to the bird when relocating the reward. This determined which cue (the location of the flap or the color of the flap) the bird had used to relocate the baited flap.

Space test. In the space version of the test, only positional information was available in phase II, because all seven flaps were identical and undecorated. Whereas the baited compartment in phase I was covered by a uniquely decorated flap, this was replaced with an undecorated flap in the same location in phase II. Thus, there were no color cues available to the bird in phase II. A bird that had relied entirely on remembering the correct color of the flaps should have searched at random, whereas a bird that had remembered the correct spatial location of the flap should have made significantly fewer looks to find the baited compartment.

Color test. For the color version of the test, none of the flaps in phase II were in the same spatial location as they were in phase I. Thus, a bird that had remembered only the spatial location of the baited compartment should have searched at random, whereas a bird that had remembered the color of the flap should have made significantly fewer looks to find the baited compartment.

Each bird was trained and tested on both versions of the task in random order but with the constraint that any one bird received an equal number of space and color trials and that it received no more than two consecutive trials of the same version. During pretraining, training, and testing, the birds were deprived of food at 8 A.M. Greenwich Mean time. Tests began at 10 A.M. Birds received one trial of each test per day.

Pretraining. During pretraining, the birds were presented with an experimental tray containing food in one of the compartments for $5 \mathrm{~min}$ daily. Three days later, once the birds were eating food from the tray, a flap was introduced to cover the baited compartment. During the second stage of pretraining, three flaps were placed on the tray. Once the birds could lift flaps to obtain a reward within 5 min, training began. Initially, food was placed under all three flaps. When a bird had learned to remove all three flaps to search for food, seven flaps were introduced. When the birds had learned to remove flaps containing food, only one site was rewarded on each trial. Trial-unique sites were introduced at this stage. Each flap was unique in terms of spatial location on the tray and in terms of a colored dot placed in the center of the flap.

Stage I: training. Training began as soon as unique flaps were introduced. At this stage, birds received a phase I followed by a phase II test. All birds received 10 space and 10 color tests. A baseline performance on each task was calculated for each bird by taking the mean performance score on the last three space and color tests.

Stage II: lesions. At the end of training, the birds were randomly divided into three groups: control, sham-lesioned, and lesioned. The surgical procedures are described below. The number of birds in each group after recovery were 10 control, 8 sham-lesioned, and 29 lesioned birds. After recovery, the birds received five space and five color trials.

Stage III: transplants. At the end of Stage II, a subset of the birds in the lesion group was randomly chosen to receive either telencephalon or $\mathrm{H}$ transplants (see below). Four to five weeks after transplantation, the birds were retested with three to five trials on the space and color tasks.

Lesion procedure. Host birds were anesthetized with equithesin (4 $\mathrm{ml} / \mathrm{kg}$ of body weight) and placed in a stereotaxic frame. The head angle was adjusted to $-3 \mathrm{~mm}$. The skull was exposed, and square sections of the skull overlying the injection sites were cut, such that the skull flap could be opened out but not removed. The following lesion coordinates were used for single, bilateral injections: $\mathrm{AP}=+1 \mathrm{~mm}$ (from the "Y" sinus); $\mathrm{L}= \pm 0.5 \mathrm{~mm} ; \mathrm{V}=-0.9 \mathrm{~mm}$. Pressure injections of $0.1 \mu \mathrm{l}$ of $10 \mathrm{mg} / \mathrm{ml}$ ibotenic acid (dissolved in $0.1 \mathrm{~m}$ sterile phosphate buffer, $\mathrm{pH}$ 7.4) per site were made using a $1 \mu \mathrm{l}$ Hamilton syringe fitted with a glass micropipette drawn to a fine tip. The pipette was left in situ for $2 \mathrm{~min}$ before withdrawal. The skull flaps were closed and the skin sutured. Shamlesioned birds were given single, bilateral injections of $0.1 \mu \mathrm{l}$ of $0.1 \mathrm{M}$ sterile phosphate buffer, $\mathrm{pH} \mathrm{7.4,} \mathrm{into} \mathrm{the} \mathrm{same} \mathrm{coordinates.} \mathrm{Normally}$ birds recovered fully in 3-4 d, after which they were behaviorally tested.

Transplantation procedure. Adult zebra finches were allowed to breed and lay eggs. The birds began to incubate the eggs once the full clutch had been laid. The day the incubation was started was considered embryonic day 1 (E1) (the incubation period was $\sim 12 \mathrm{~d}$ ). Because the optimal donor age for harvesting the fetal tissue was unknown, different groups of lesioned birds were given transplants of fetal $\mathrm{H}$ and $\mathrm{AT}$ tissue taken from donors at two embryonic ages. Alvarez-Buylla et al. (1994) showed that the greatest numbers of $\mathrm{H}$ cells are born at $\sim \mathrm{E} 9$ and that the AT has a similar rate of development at E9 as at E5. Thus, the host birds allocated to receive either AT or $\mathrm{H}$ transplants were randomly subdivided further into two additional groups: one group from each received transplants of tissue taken from E6-E7, and the other group received transplants of tissue taken at E9-E10. The H and AT tissue was taken from the same embryos and treated in the same manner for transplantation. The embryonic dissection and transplantation procedures for cell suspensions were similar to those described for the rat in Dunnett and Björklund (1992) and Björklund and Dunnett (1992). All procedures, including harvesting of donor tissue, preparation of the cell suspension, and grafting surgery, were conducted under aseptic conditions. The surgical instruments and all materials were sterilized before use. The embryos were removed from the eggs and transferred to a sterile dish containing sterile $0.6 \%$ glucose $+0.75 \%$ saline (GS). After decapitation, the brain was excised from the skull, and the overlying meninges were removed. Embryonic $\mathrm{H}$ tissue was taken from the dorso-caudal part of the brain, and telencephalon tissue was taken from the rostral part of the same brain (Fig. 1). The respective tissue pieces were collected in sterile Eppendorff tubes containing GS and mechanically dissociated using Pasteur pipettes without trypsin treatment. After centrifugation and withdrawal of supernatant, fresh GS solution was added, and the tissue was gently dissociated further. The solution was centrifuged and the supernatant was withdrawn, and $5 \mu \mathrm{l}$ of GS per dissected tissue piece was then added to make up the final cell suspension for injection. The tissue was not fully dissociated and consisted of cell aggregates small enough to be injected using a $1 \mu \mathrm{l}$ Hamilton syringe connected to a glass pipette drawn to a fine tip. Between injections, the cell suspension was kept on ice, and the cell viability was assessed to be between 80 and $90 \%$ (according to the method described in Björklund and Dunnett, 1992). Typically, H and AT tissue from three or four embryos of the same clutch was pooled to obtain $\sim 100,000 \mathrm{cells} / \mu \mathrm{l}$ for injection. Transplantation was performed $8-10 \mathrm{~d}$ after lesioning, because mammalian transplantation studies have suggested that graft survival is optimal $8-10 \mathrm{~d}$ after lesioning (NietoSampedro et al., 1984; Stein and Mufson, 1987; Nieto-Sampedro, 1988). For transplantation, the surgical procedures used for lesioning were basically repeated, i.e., the anesthetized birds were placed in the stereotaxic frame, and the skull flaps were reopened. Slow (for $\sim 2 \mathrm{~min}$ ) bilateral pressure injections of $1 \mu \mathrm{l}$ of cell suspension per site were made into the $\mathrm{Hf}$ at the same coordinates as those used for lesions. The pipette was then left in situ for 2 min before withdrawal. The skull flaps were closed and the skin sutured.

Histology. At the end of behavioral testing, 5-6 weeks after transplan- 


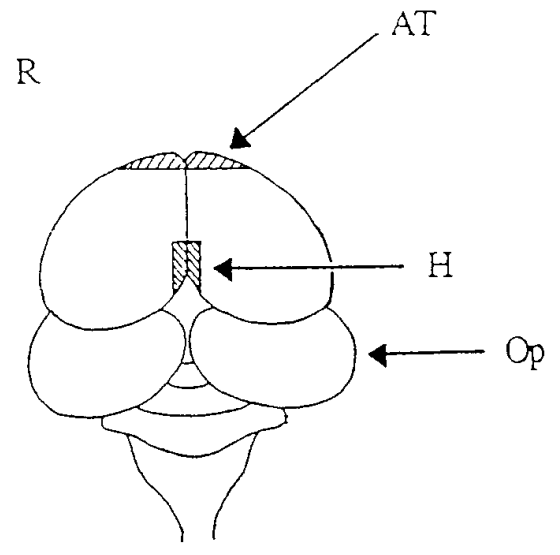

$\mathrm{C}$

Figure 1. Schematic diagram of an E9-E10 zebra finch embryo, dorsal view. The shaded areas, approximately, show the dorsal hippocampal $(H)$ and anterior telencephalon $(A T)$ regions, which were dissected out and collected separately for transplantation. $O p$, Optic lobe; $R$, rostral; $C$, caudal.

tation, the birds were given a lethal overdose of sodium pentobarbitone and perfused intracardially with $30 \mathrm{ml}$ of PBS, followed by $4 \%$ buffered paraformaldehyde. The brains were removed and kept in the same fixative for $24 \mathrm{hr}$, after which they were left in $25 \%$ sucrose until they sank. Cryostat sections were cut at a thickness of $25 \mu \mathrm{m}$, and a 1:5 series of sections were processed for Nissl staining. Selected sections were also stained immunohistochemically with antibodies to parvalbumin (PA, Sigma, Poole, UK) to the $200 \mathrm{kDa}$ neurofilament protein phosphorylated and nonphosphorylated (NFP, Sigma), and substance P (SP, Medicorp). These antibodies were used to identify the presence of neurons and fibers within the transplants. The dilutions used were 1:1000 for PA, 1:400 for NFP, both diluted in $0.3 \%$ Triton X-100 in PBS, and 1:500 for SP in PBS. After treatment of the sections in $1 \%$ hydrogen peroxide in methanol for 10 min, the sections were washed in PBS and blocked with normal serum (rabbit or horse) for $30 \mathrm{~min}$. Primary antibody was applied, and the sections were left overnight (at $4^{\circ} \mathrm{C}$ ). After 3 washes in PBS, the sections were left for $30 \mathrm{~min}$ in the secondary antibody (anti-mouse for PA and NFP, anti-rat for SP), and then incubated in avidin-biotin solution (ABC kit, Vector Laboratories) for $30 \mathrm{~min}$. The chromogen solution was applied for $4-6 \mathrm{~min}$ and consisted of $0.025 \%$ diaminobenzidine $+0.03 \%$ hydrogen peroxide in PBS. The sections were washed, dehydrated, and mounted in Eukitt.

Volume measurements. A video camera connected to a Leica light microscope was used to capture the image of the brain sections into an Image 1.42 program in a Macintosh computer to measure the areas of the lesions within the Hf, the areas of the transplants, and the areas of the hippocampus for calculation of the estimated total lesion volume, total transplant volumes, and total Hf volume (according to the method in Krebs et al., 1989; Clayton and Krebs, 1994). The latter will be an approximate estimate because of the distortion of the Hf after lesioning and transplantation.

All measurements and behavioral testing were performed "blind." Statistics used were Student's $t$ test and ANOVA with or without repeated measures. The HSD test for two group comparisons of means in a repeated-measures design (Hays, 1988) and Duncan's New Multiple Range (NMR) test were used for post hoc tests.

\section{RESULTS}

\section{Histology of the lesions}

The total volume of the lesions from both hemispheres in the lesion-only (L) group of birds varied from 0.045 to $0.218 \mathrm{~mm}^{3}$ (mean $\left.=0.106 \pm 0.059 \mathrm{~mm}^{3}, \mathrm{SD}\right)$. This represents $\sim 0.64-2.9 \%$ $($ mean $=1.67 \pm 0.791 \%)$ of the estimated total Hf volume. There was damage neither to the hyperstriatum accessorium nor to the underlying hyperstriatum ventrale, and apart from a small needle tract, there was no Hf damage in the sham-lesioned birds. (Where damage in the sham-lesioned birds was observed, it was limited to a maximum of $0.04 \%$ of $\mathrm{Hf}$ volume and appeared to be attributable to insertion of the needle.)

There was no evidence of an inflammatory reaction, necrosis, or calcified tissue. Nissl-stained sections showed a loss of neuronal cell bodies within the lesion site (Fig. $2 A, B$ ), and anti-PA staining of selected sections revealed a loss of PA-positive neurons in the lesion area (Fig. 2C,D) (a quantitative analysis of the loss of PA-positive neurons will be presented elsewhere). The location of the lesions within the Hf varied among birds; three had lesions of the dorsomedial Hf; four had lesions of the lateral Hf; and one had lesions of the medial Hf. The behavioral performance of the lesioned birds was poor regardless of the locations of the lesions.

\section{Histology of the transplants}

The birds that received $\mathrm{H}$ tissue $(n=6)$ taken from E9-E10 donors all had surviving transplants, whereas those that received E6-E7 tissue did not have visible $\mathrm{H}$ transplants $(n=5)$. Three of four birds that had received AT tissue from E9-E10 donors and two of five birds that had received AT tissue from E6-E7 donors had visible transplants. Therefore, the donor age was critical for the survival of the transplanted $\mathrm{H}$ tissue, but it appeared to be less important for the survival of AT tissue.

All birds with surviving $\mathrm{H}$ and AT transplants had several small transplants or cell aggregates scattered within different areas of the ventricle. In these birds, all of the $\mathrm{H}$ transplanted birds and three of five of the AT transplanted birds had grafts that were growing partly within the Hf and partly within the underlying ventricle (Fig. 3). These transplants appeared to be joined with the host Hf but did not show obvious transplant-host fiber connections by immunostaining with the $200 \mathrm{kDa}$ NFP (Fig. $4 A$ ) and with SP-antibody (Fig. 4B). These antibodies revealed the presence of both unbeaded and beaded fibers, respectively, within the transplants. The latter staining is characteristic of SP-labeled beaded fibers within the dorsal hippocampus of adult birds (Erichsen et al., 1994).

The surviving transplants appeared healthy, with little evidence of necrosis or an immune reaction (identified by the absence of lymphocytes, perivascular cuffing, or macrophages in Nissl preparations). Immunohistochemical staining of selected sections with anti-PA, a neuron-specific antibody (Celio and Heizmann, 1981), showed that the $\mathrm{H}$ and AT transplants had PA-positive neurons that were of the same size and type as those of the surrounding host brain and with visible processes (Fig. 4C). Blood vessels and erythrocytes were also found in the intraventricular transplants (Fig. 4D). One of the blood vessels appeared to be forming a bridge between the intraventricular transplant and the host parenchyma (Fig. $4 B$ ), indicating that the transplants had probably received blood supply from the host brain.

The mean of the combined volumes of the AT transplants from the five birds with surviving telencephalon transplants was $0.156 \pm$ $0.091 \mathrm{~mm}^{3}$ (SD) and that of the $\mathrm{H}$ transplants from six birds with surviving $\mathrm{H}$ transplants was $0.075 \pm 0.017 \mathrm{~mm}^{3}$. This difference was not significant [Student's $t$ test $(9)=-1.96$, NS].

\section{Behavioral performance}

The performance of the birds on the space and color tasks was tested during each of the three stages of the experiment. The birds were trained during stage I. During stage II, the birds were divided into three groups: a control group $(\mathrm{C}, n=10)$, sham- 

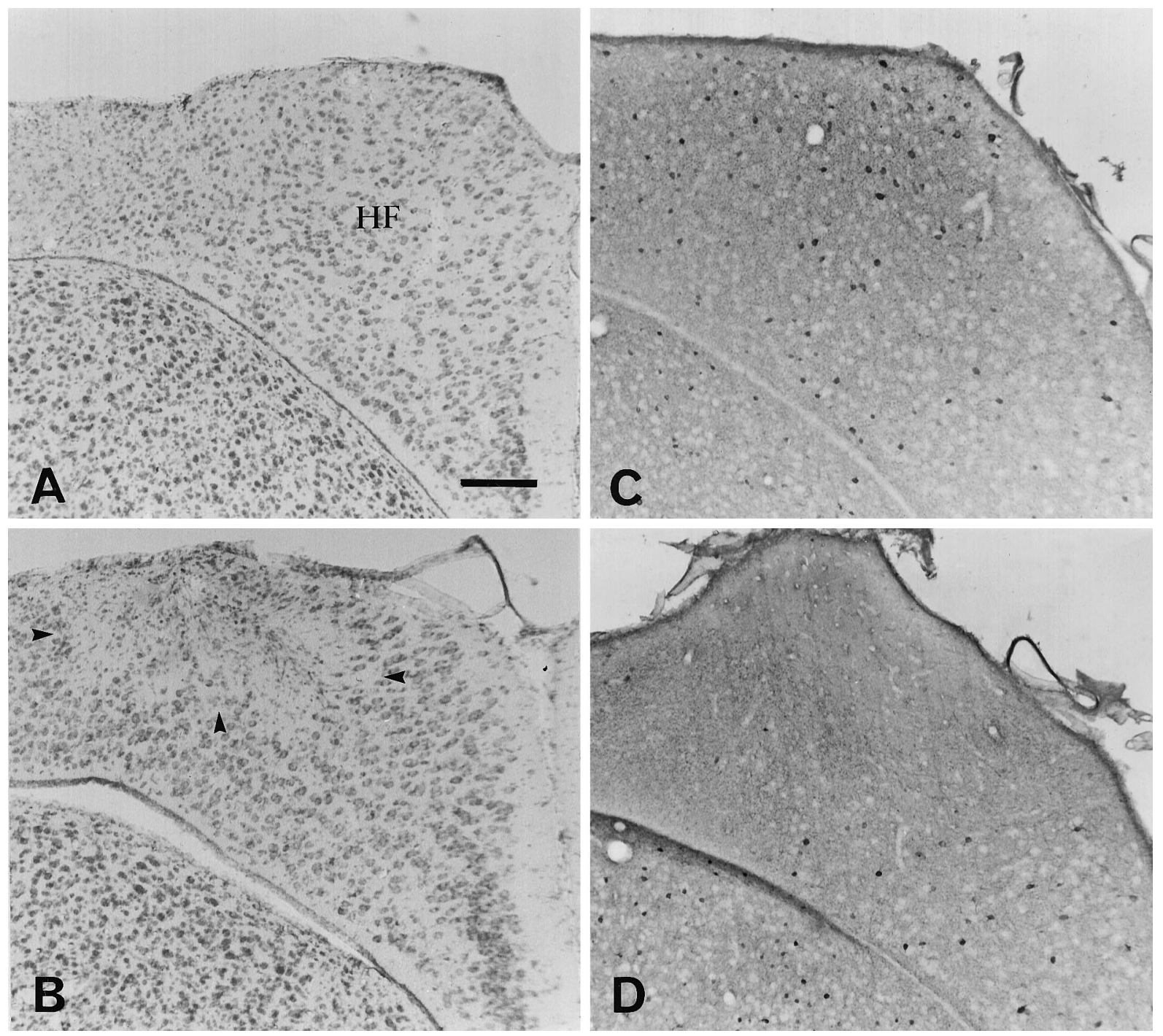

Figure 2. Hippocampal lesions are shown here with Nissl staining and anti-PA immunohistochemistry. The appearance of the hippocampal formation $(H F)$ in the normal brain and the lesioned brain with Nissl staining is shown in $A$ and $B$, respectively. The arrowheads in $B$ show the approximate boundaries of the lesion. The lesioned area was characterized by a loss of neuronal cell bodies, as shown with immunohistochemistry with anti-PA antibody: normal staining is shown in $C$, and the loss of PA-positive cells in the hippocampus is shown in $D$. This lesioned area is primarily located within the dorsolateral hippocampus and extends a little into the area parahippocampalis. The sections used for immunohistochemistry shown here were not directly adjacent to those used for Nissl staining. Scale bar: $A-D, 128 \mu \mathrm{m}$.

lesion group (S, $n=8)$, and ibotenic acid- lesioned group (L, $n=$ 29). During stage III, the control and sham-lesion groups remained without additional treatment, and the lesioned birds were randomly subdivided into three treatment groups: lesion-only (no additional treatment), lesion with $\mathrm{H}$ transplants, and lesion with telencephalon transplants. The histological examination at the end of the experiment showed that some of the transplanted birds did not have visible transplants (discussed above). Therefore, the transplanted groups were subdivided into four additional subgroups: $\mathrm{H}$ transplanted birds with visible transplants $(\mathrm{H}-\mathrm{T})$ and without visible transplants (H-NT) and telencephalon transplanted birds with visible transplants (AT-T) or without visible transplants (AT-NT). This categorization was performed for the statistical analysis only, and it did not preclude the possibility that there were undetected transplants growing in the NT groups or that the transplants in this group had died just before the end of the experiment. The advantage of this subdivision was that it controlled for any behavioral effects of two surgical procedures: the birds with nonsurviving transplants served effectively as "sham-transplanted" groups, because all transplanted birds had received lesions and transplants in two separate surgical operations. Thus, in stage III, there were seven groups of birds: C, S, L, H-T, H-NT, AT-T, and AT-NT ( $n=10,8,9,6,5,5$, and 4, respectively). Data on space and color performance were available for each bird at all three stages of the experiment. The following comparisons can be made.

\section{Space and color performance compared in stages I and II}

There were no significant differences between the performances of the birds in the space and color tasks in stage I (during training) $\left(F_{1,46}=0.007, \mathrm{NS}\right)$. There was no correlation between the space and color performances of the birds in stage I (Spearman's $\rho=$ -0.051 ) showing that the performance on the space version of the task was unrelated to that on the color version of the task. In stage II, after lesions, there were significant effects of treatment $(\mathrm{C}, \mathrm{S}$, or L; by ANOVA, $F_{2,44}=36.06, p<0.0001$ ), task type (space or 


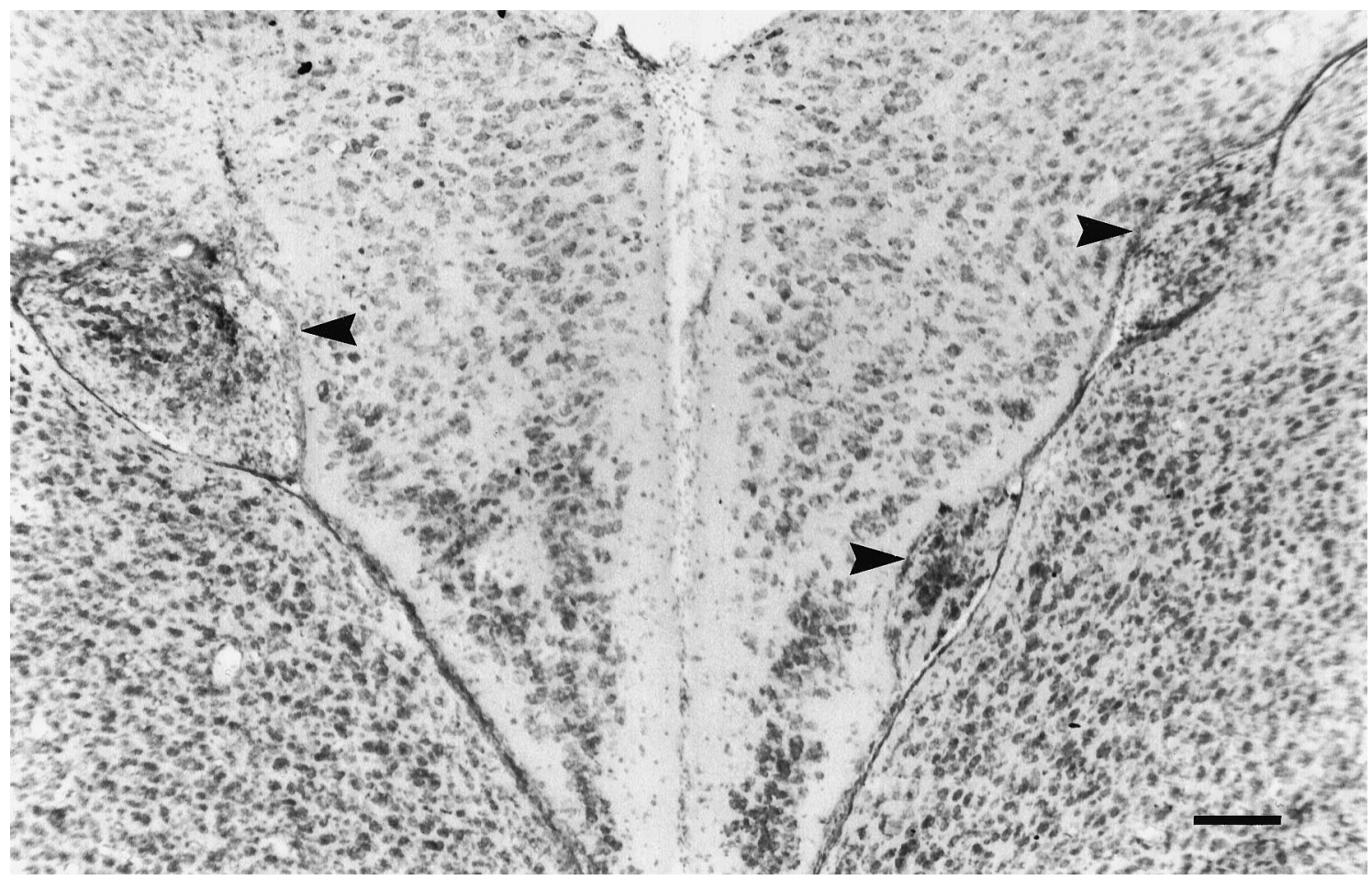

Figure 3. The typical location of some of the transplants. Three transplants (arrowheads) are shown growing partly within the ventricle underlying the $\mathrm{Hf}$ and partly within the $\mathrm{Hf}$ itself. The needle tract is visible on the right $\mathrm{Hf}$ above the transplant (Nissl staining). The arrowheads are placed on the side of the Hf. Scale bar, $112 \mu \mathrm{m}$.

color) $\left(F_{1,44}=4.55, p<0.05\right)$, and the interaction term $\left(F_{2,44}=\right.$ $15.73, p<0.0001)$ but no effects of sex. For the space task, the postlesion performance of the lesioned birds (stage II) was significantly poorer than the prelesion performance $(p<0.05$, Tukey's HSD), and it was significantly worse than the $\mathrm{C}$ and $\mathrm{S}$ groups $(p<0.05$, Duncan's NMR) (Fig. $5 A)$. For the color task, the postlesion performance of the lesioned birds (stage II) was significantly poorer than the prelesion performance $(p<0.05$, Tukey's HSD), and it was significantly worse than the $\mathrm{C}$ and $\mathrm{S}$ groups ( $p<0.05$, Duncan's NMR) (Fig. 5B). However, the prelesion performance of the lesioned birds on the color task was also significantly better than that for the $\mathrm{C}$ and $\mathrm{S}$ groups $(p<$ 0.05, Duncan's NMR) (Fig. 5B).

\section{Overall before and after comparisons for all stages: I, II, and III}

A comparison of all groups of birds before and after lesions and transplants (for all three stages) was performed individually for the space and color data. A two-factor ANOVA with repeated measures on stage (I, II, and III) and with treatment and sex as between-subject factors was performed for mean number of looks in the space or color task.

Space task. For the space task, there was a significant effect of treatment $\left(F_{6,34}=26.7, p<0.0001\right)$ but no effect of $\operatorname{sex}\left(F_{1,34}=\right.$ 2.17 , NS) or interaction $\left(F_{5,34}=1.16, \mathrm{NS}\right)$; there were also significant effects of stage $\left(F_{2,68}=18.57, p<0.0001\right)$ and stage by treatment interaction effects $\left(F_{2,68}=5.67, p<0.0001\right)$. Figure $6 A$ shows the performance of the birds in the space task at each of the three stages of the experiment. The following comments can be made about this data. (1) The control and sham birds did not differ in space performance among the three stages of the experiment, showing that the different stages of the experiment did not have a significant effect on the performance of the birds per se. (2) The mean stage I performance of the birds in all groups did not significantly differ among the groups (one-factor ANOVA: $F_{6,40}=$ 1.004 , NS), showing that the initial performance of the birds before surgery for lesions and transplants was the same for all groups of birds. (3) The lesioned-only (L) birds showed a significant performance deficit after the lesion in stage II that persisted into stage III ( $p<0.05$, by HSD), showing that the lesioned birds did not spontaneously recover in stages II or III. (4) The performances of the two telencephalon transplanted groups (AT-T and AT-NT) were similar to those of the lesion-only group; there was a significant drop in performance after the lesion (in stage II, especially in the AT-T group) that persisted into stage III, after the transplantation, showing that the telencephalon transplants did not ameliorate the performance deficits. (5) There were significant differences in space performance between stages I and II and between stages II and III in the $\mathrm{H}$ transplanted birds with visible transplants $(\mathrm{H}-\mathrm{T})$, showing that a behavioral deficit was produced after the lesion in stage II, which was reversed by the transplants in stage III. (6) There were no significant differences in performance between stages I, II, and III in the H transplanted birds without visible transplants (H-NT). Although the repeatedmeasures analysis showed that there was no significant effect of lesion in this group, the between-groups analysis did reveal a significant effect of lesion compared with the $\mathrm{C}$ and $\mathrm{S}$ groups, and transplantation did not improve performance significantly compared with the $\mathrm{C}, \mathrm{S}$, and $\mathrm{H}-\mathrm{T}$ groups (see below).

These conclusions were supported further by one-way ANOVAs on space performance between the groups in stages II and III; for stage II, the $\mathrm{C}$ and $\mathrm{S}$ groups differed significantly from all of the other lesioned groups (L, H-T, H-NT, AT-T, and AT-NT), 

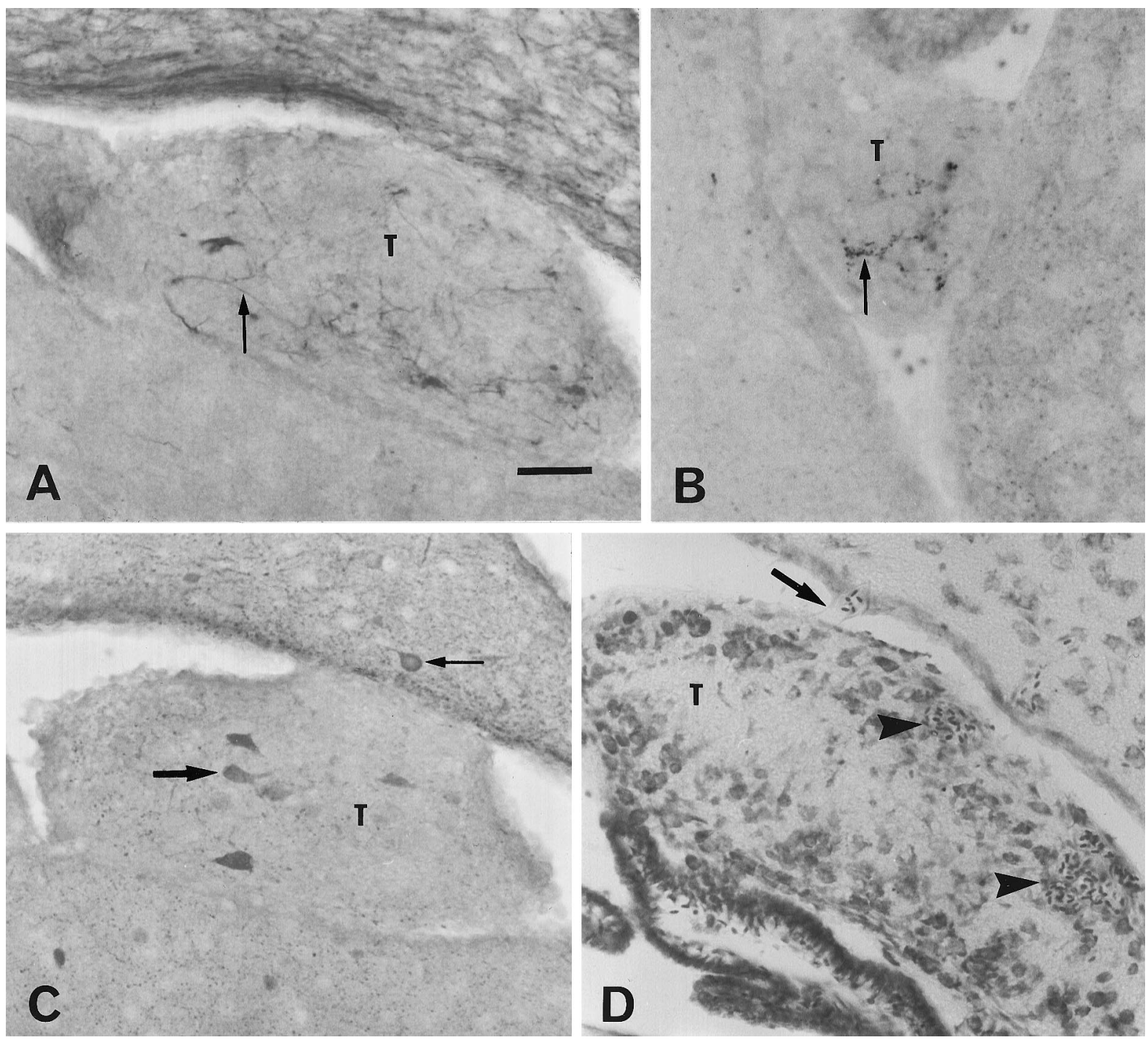

Figure 4. The typical histological characteristics of the $\mathrm{H}$ and AT transplants $(T)$ located in the ventricles. These intraventricular transplants contained both unbeaded $(A)$ and beaded fibers $(B)$ as shown by immunohistochemistry with antibodies to a $200 \mathrm{kDa}$ NFP and to SP, respectively (arrows show labeled fibers within the transplants). $C$, PA immunohistochemistry identified neurons within the transplants that had dendritic processes emanating from the cell bodies (large arrow). These neurons were similar in size and shape to those of the host brain (small arrow). D, Blood vessels and clusters of erythrocytes within blood vessels (arrowheads) were present in the transplants (Nissl staining). One blood vessel appeared to be forming a bridge between the graft and the host (large arrow), indicating that the transplants may have received host blood supply. These transplants were located in different parts of the ventricle; those shown in $A$ and $C$ were located in the lateral ventricle between the hippocampus and the hyperstriatum ventrale, and those shown in $B$ and $D$ were located in the ventral part of the ventricle. Scale bars: $A, C, D, 37 \mu \mathrm{m} ; B, 28 \mu \mathrm{m}$.

as shown by a significant $\operatorname{ANOVA}\left(F_{6,40}=13.26, p<0.0001\right)$ and post hoc means comparisons ( $p<0.05$, Duncan's NMR). For stage III, the $\mathrm{C}, \mathrm{S}$, and $\mathrm{H}-\mathrm{T}$ groups differed significantly $(p<$ 0.05) from the other groups (L, H-NT, AT-T, and AT-NT).

Color task. For the color task on within-factor comparison among the stages I, II, and III; there were no significant effects of treatment $\left(F_{6,34}=1.51, \mathrm{NS}\right), \operatorname{sex}\left(F_{1,34}=2.18, \mathrm{NS}\right)$, or stage $\left(F_{2,68}\right.$ $=0.868, \mathrm{NS})$. There was a significant effect of stage by treatment interaction $\left(F_{2,68}=2.06, p<0.05\right)$ (Fig. $\left.6 B\right)$. The lesion (L) group showed the only significant difference between stage I and stages II and III ( $p<0.05$, Tukey's HSD). However, in a between-factor comparison of stage III, the lesion group did not significantly differ from the control $(\mathrm{C})$ and sham-lesion $(\mathrm{S})$ groups $\left(F_{2,44}=\right.$ 0.113 , NS), and there were no other significant differences between the stages or among the other groups (Fig. 6B). Performance on the color task was not affected by transplantation.

\section{DISCUSSION}

The results showed that (1) small bilateral excitotoxic lesions of the Hf produced significant performance deficits on the space task; (2) depending on the age of the donor, both fetal $\mathrm{H}$ and telencephalon tissue had survived and grown within the ventricles and some also partly within the Hf of the bird brain; (3) these transplants were healthy and contained neuronal and axonal elements but did not appear to have transplant-host interconnections; (4) lesioned birds with visible $\mathrm{H}$ transplants showed significant behavioral recovery on the space task but not on the color task; (5) lesioned birds with telencephalon transplants or without visible, presumably nonsurviving, $\mathrm{H}$ or telencephalon transplants did not show significant behavioral recovery on either the space or the color tasks; and (6) the size and placement of the lesions and transplants were not impor- 

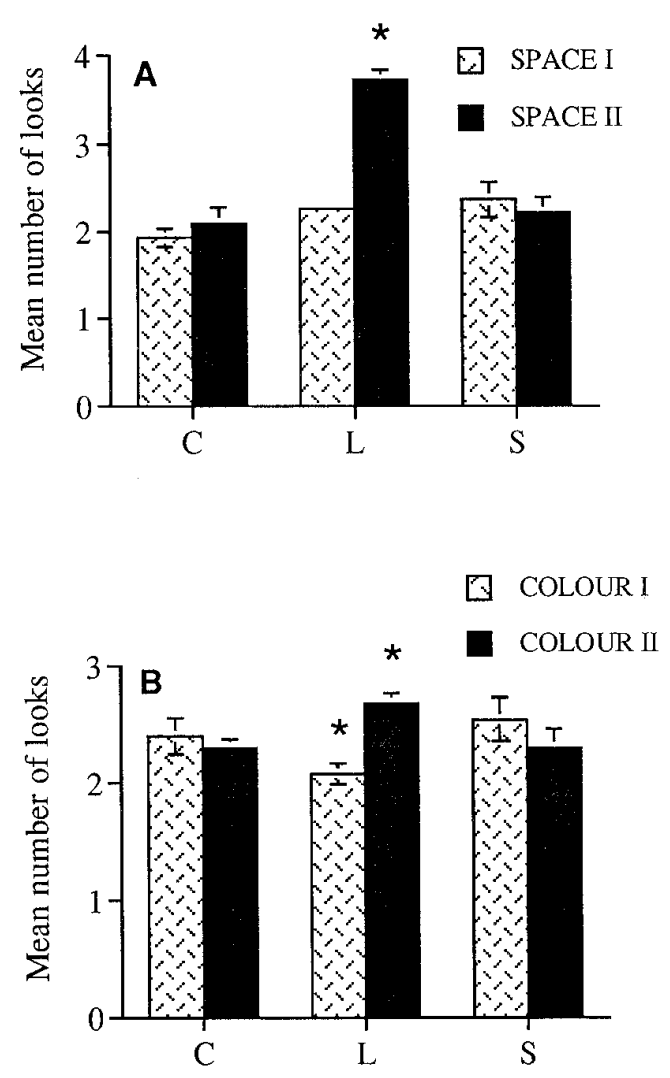

Figure 5. Performance of the birds on a one-trial associative memory task, as measured by the mean number of looks to find the baited compartment, before lesion in stage I (baseline performance) and after lesion in stage II. Performance at chance level $=4$ looks; fewer looks indicate better performance. $A$, For the space task, the lesioned $(L)$ birds were significantly worse compared with the prelesion performance and the control $(C)$ and shamlesioned $(S)$ birds $(* p<0.05)$. The baseline performance (stage I) did not differ among groups. $B$, For the color task, the baseline color performance (stage I) of the L birds was significantly better than that of $\mathrm{C}$ and $\mathrm{S}$ birds, and the postlesion color performance (stage II) of the L birds was significantly worse than the $\mathrm{C}$ and $\mathrm{S}$ birds $\left({ }^{*} p<0.05\right)$.

tant factors governing the extent of behavioral impairment or recovery, respectively.

This is the first lesion and transplantation study in adult birds that shows significant spatial memory impairments produced by small ibotenic acid lesions of the avian hippocampus and a successful reversal of these spatial memory deficits by neural transplantation of homologous $\mathrm{H}$ tissue, but by neither heterologous telencephalon tissue nor neural transplantation per se. The disruption of performance on behavioral tasks involving spatial information processing by aspiration lesions of the avian Hf has been demonstrated previously (Sherry and Vaccarino, 1989; Sherry et al., 1992) and is supported here with smaller, excitotoxic lesions of the Hf. Compared with aspiration lesions, which resulted in the removal of $\sim 37 \%$ of the total volume of the hippocampus (Sherry and Vaccarino, 1989), together with some damage to extra-Hf tissue, the excitotoxic lesions in this study were localized to the $\mathrm{Hf}$ and only removed up to $2.9 \%$ of the $\mathrm{Hf}$, while still producing significant effects on behavioral performance. Thus, even a relatively small disruption of the normal Hf cytoarchitecture can have significant functional consequences. Previous studies have shown that the lesioned area, which was primarily the dorsal region of the hippocampus, normally has a concentration of many different cell types of different neurochemical composition
(Erichsen et al., 1991, 1994). The behavioral impairments may not be related to the size of the lesions per se but may instead be related to the loss of neurons of specific type(s) and/or specific neurochemical composition. For example, neurons containing $\mathrm{PA}$, which is a calcium-binding protein, appear to have been destroyed by the lesions (Fig. $2 D$ ). A thorough assessment of the nature and extent of the lesions and a characterization of the types of cells that may have been lost or damaged by the lesions and their neurochemical composition are therefore required, and this result should form the basis of future experiments.

Previous studies had found no effect of $\mathrm{Hf}$ aspiration lesions on color discrimination tasks (Sherry and Vaccarino, 1989), whereas a significant effect of lesions on color performance compared with prelesion performance of the birds was observed here. However, of the five lesioned groups in this study (L, H-T, H-NT, AT-T, and AT-T), only the lesion-only (L) group showed a significant effect of lesion on color performance compared with prelesion performance. However, because the birds were assigned randomly to each of the groups, the lesion-only group may have been assigned, by chance, birds that showed good prelesion performance on the color discrimination task. Therefore, it is possible that this experiment may have revealed a novel effect of small excitotoxic lesions of the hippocampus on performance on a color discrimination task, which may be dependent on good prelesion behavioral performance of the birds. That a previous study (Sherry and Vaccarino, 1989) had failed to find such an effect may be attributable to several differences between the two studies, including the species used, the type and size of the lesions, and differences in the behavioral paradigms.

The intraventricular $\mathrm{H}$ and telencephalon transplants grew either in the ventricle adjacent to and partly within the Hf and/or as small cell aggregates in other parts of the ventricles. This type of growth had probably occurred because the injected cell suspension contained tissue that was not fully dissociated; trypsin was not used in this study, because previous pilot attempts resulted in poor cell viability. The pressure injections into the hippocampus may have forced the injected cells into the underlying ventricle, thereby dispersing clumps of embryonic cells throughout the ventricle, resulting in the growth of cell aggregates in different parts of the ventricles. Immunohistochemical staining with antibodies to the $200 \mathrm{kDa}$ NFP, SP, and PA showed that both types of transplants had neurons (Fig. $4 C$ ) and nerve fibers of both the beaded and unbeaded varieties (Fig. $4 A, B)$. The transplants appeared healthy and seemingly received blood supply from the host brain (Fig. $4 D$ ). In addition, the optimal donor age for the harvesting and transplantation of $\mathrm{H}$ tissue was found to be E9-E10, because the birds that received tissue from embryos at an earlier stage (E6-E7) did not have visible, surviving transplants. Other donor ages were not tested in this initial study. The donor age for transplantation of telencephalic tissue did not appear to be critical, because surviving transplants were found in birds that were grafted with tissue taken from different donor ages, although survival appeared to be better with E9-E10 tissue. The birds that had nonsurviving transplants of either type served as ideal controls in this experiment, because they were subjected to all the surgical, lesioning, and transplantation procedures that the other birds with surviving transplants had received. Thus, the results clearly showed that the transplant-induced behavioral recovery was dependent on the presence and type of transplant and not on any nonspecific effects of surgical or transplantation procedures per se.

Transplant-mediated behavioral improvements have been reported in many mammalian studies, but the mechanism(s) by which these occur is still largely speculative. Some of the proposed 
Figure 6. The performance of the birds (mean number of looks) in each group before lesions (stage I), after lesions (stage II), and after transplantation (stage III). $A$, For the space task, the significant differences in the various groups among stages I, II, and III are shown in the graph $\left({ }^{*} p<0.05\right.$, Tukey's HSD for "within-bird" comparisons). The controls $(C)$ and sham-lesioned $(S)$ birds performed better than chance (of 4 looks) during all three stages. After lesioning (stage II), the lesioned $(L)$ birds performed at or near chance level and significantly worse than their prelesion performance. After transplantation, only the birds with surviving $\mathrm{H}$ transplants $(H-T)$ showed significant behavioral recovery, effectively reversing the lesioninduced deficit; compare stage I with II and stage II with III $\left({ }^{*} p<0.05\right.$, HSD). The $\mathrm{H}$ transplanted birds without visible transplants $(H-N T)$ did not show any significant differences among the three stages. The telencephalon transplanted birds with transplants $(A T-T)$ or without visible transplants $(A T-N T)$ only showed a significant effect of lesion but no improvement after transplantation. $B$, The mean number of looks on the color task for each group, among stages I, II, and III. Only the lesioned birds showed a significant performance impairment after lesioning $\left({ }^{*} p<0.05\right.$, HSD).
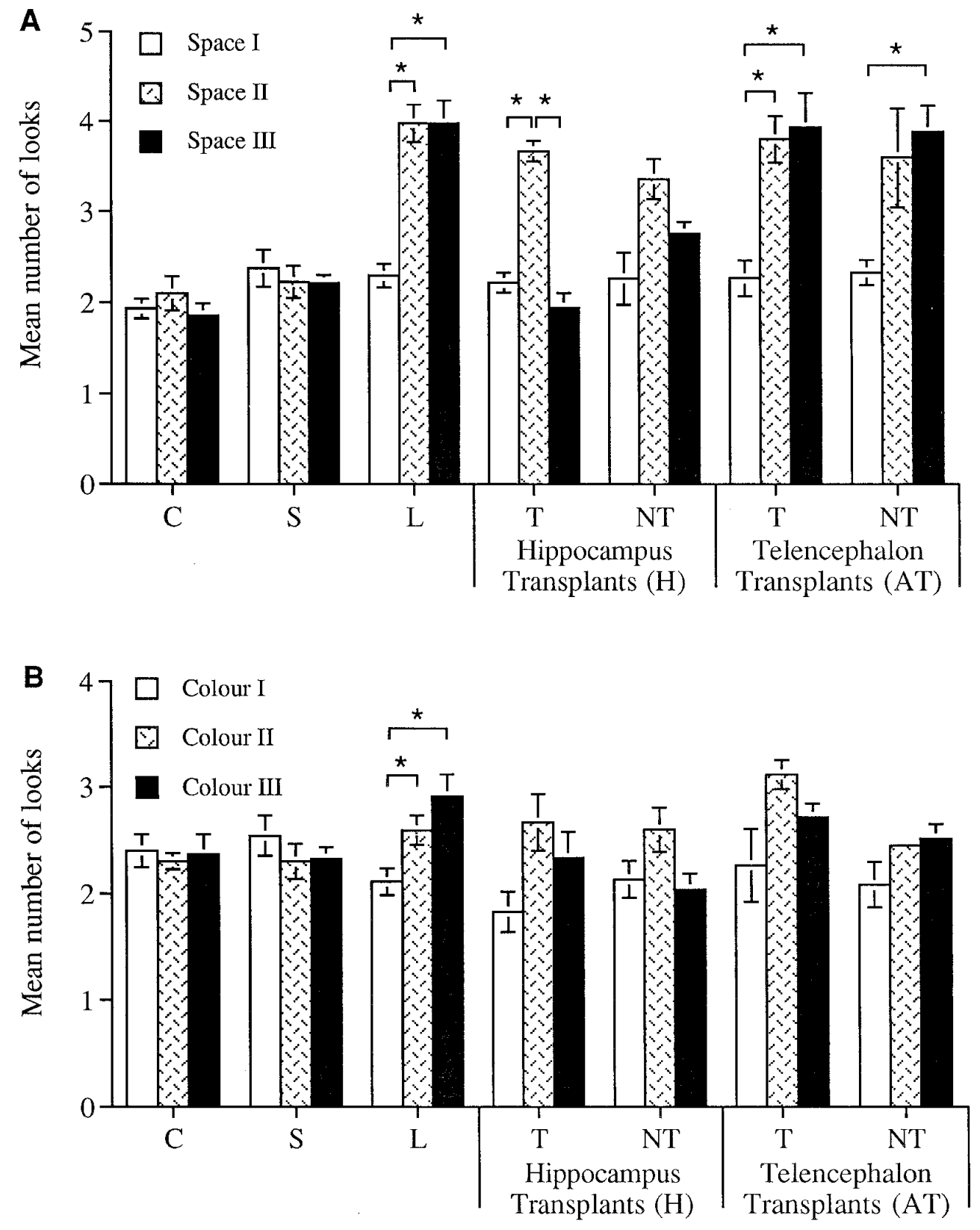

mechanisms of recovery include the release of trophic or other factors that promote host neuronal repair or growth, replacement of the lost cells, and formation of graft-host interconnections leading to the reestablishment of the lesioned pathways and the release of neurotransmitters either specifically at the newly formed synaptic terminals or diffusely into the host brain (for review, see Gage and Fisher, 1991; Nishino, 1993; Sinden et al., 1995; Stein and Glasier, 1995). Here, because most of the visible $\mathrm{H}$ transplants were located in the ventricle, without any obvious transplant-host connections with the host brain, it is likely that the transplant-mediated functional recovery was attributable to the release of trophic factors or neurochemical substances or other factors from the transplants into the ventricles, which may have either promoted host brain repair or may have had a general stimulating effect on the host. Therefore, it would be interesting to study the behavioral effects of the removal of successful transplants in future experiments.
The importance of the trophic hypothesis of graft function is now well known. For example, Stein and colleagues (Labbe, 1983; Stein, 1988) showed that lesions of the frontal cortex in adult rats lead to an impairment in a delayed spatial alternation T-maze task (DSA). Rats with transplants of E19 frontal cortex tissue into the lesioned cortex showed significant recovery in DSA, compared with lesion-only controls, $5 \mathrm{~d}$ after the operation. Such rapid recovery could be attributed to the release of trophic or other factors from the transplants into the host rather than through the formation of new transplant-host interconnections, which would not occur so rapidly (Labbe, 1983; Stein, 1988; Stein and Glasier, 1995). In an additional supporting study, Stein (1988) removed the transplants in one group of rats and found that the performance on DSA 3 months after surgery was not impaired in these rats. Woodruff et al. (1990) had similar findings, but in an $\mathrm{H}$ lesion model; $\mathrm{H}$ transplanted rats showed better performance in a barpress withholding response (DRL-20) compared with lesion-only 
rats, and removal of the $\mathrm{H}$ transplants did not lead to behavioral impairment. These studies suggest that the continued presence of the transplant was not necessary for the maintenance of the behavioral recovery. Therefore, neither the formation of grafthost interconnections nor the continued release of neurotransmitters by the transplants did not appear to be mechanisms by which behavioral function had been restored. Instead, it was suggested that trophic factors may have been released by the grafts or that glial cells of the transplants may have acted to remove injuryinduced excitotoxins or had stimulated the release of trophic factors from host cells leading to host brain repair (Stein, 1988; Woodruff et al., 1990; Stein and Glasier, 1995).

If trophic or other factors were released by the transplants, it is evident that they were specifically released by the $\mathrm{H}$ transplants and not by telencephalon transplants. However, in this initial study, it was not possible to perform additional examination of the transplants to determine the differences, if any, in neurochemical composition between the $\mathrm{H}$ and telencephalon transplants that may help explain the differences in the transplant-mediated recovery of function. This study also could not disprove the existence of graft-host interconnections or the presence of unidentified transplanted cells actually growing within the host hippocampus or another brain region. However, this is the first experiment showing that neural transplants can survive in the adult bird brain and that the transplants can have specific functional effects. In addition, the results provide additional support for the involvement of the avian hippocampus in learning and memory formation. Therefore, this study provides a new paradigm for the further examination of the structure-function relationships of defined regions of the bird brain.

\section{REFERENCES}

Alvarez-Buylla A, Ling C-Y, Yu WS (1994) Contributions of neurons born during embryonic, juvenile, and adult life to the brain of adult canaries: regional specificity and delayed birth of neurons in the songcontrol nuclei. J Comp Neurol 347:233-248.

Auer RN, Jensen ML, Whishaw IQ (1989) Neurobehavioural deficit due to ischemic brain damage limited to half the CA1 sector of the hippocampus. J Neurosci 9:1641-1647.

Bingman VP (1990) Spatial navigation in birds. In: Neurobiology of comparative cognition (Olton DS, Kesner RP, eds), pp 423-447. Hillsdale, NJ: Erlbaum.

Bingman VP (1993) Vision, cognition and the avian hippocampus. In: Vision, brain and behaviour in birds (Ziegler HP, Bischoff H-J, eds), pp 391-408. Cambridge, MA: MIT.

Björklund A, Dunnett SB (1992) Neural transplantation in adult rats. In: Neural transplantation: a practical approach (Dunnett SB, Björklund A, eds), pp 57-78. New York: Oxford UP.

Celio MR, Heizmann CW (1981) Calcium-binding protein parvalbumin as a neuronal marker. Nature 293:300-302.

Clayton NS, Krebs JR (1994) Hippocampal growth and attrition in birds affected by experience. Proc Natl Acad Sci USA 91:7410-7414.

Davis HP, Volpe BT (1991) Memory performance after ischemic or neurotoxin damage of the hippocampus. In: Symposia medica hoechst: the biology of memory (Squire LR, Lindenlaub E, eds), pp 477-507. New York: Schattauer.

Dunnett SB, Björklund A (1992) Staging and dissection of rat embryos. In: Neural transplantation: a practical approach (Dunnett SB, Björklund A, eds), pp 1-18. New York: Oxford UP.
Eichenbaum H (1996) Is the rodent hippocampus just for "place"? Curr Opin Neurobiol 6:187-195.

Erichsen JT, Bingman VP, Krebs JR (1991) The distribution of neuropeptides in the dorsomedial forebrain of the pigeon (Columba livia). J Comp Neurol 314:478-492.

Erichsen JT, Ciocchetti A, Fontanesi G, Bagnoli P (1994) Neuroactive substances in the developing dorsomedial telencephalon of the pigeon (Columba livia): differential distribution and time course of maturation. J Comp Neurol 345:537-561.

Gage FH, Fisher LJ (1991) Intracerebral grafting: a tool for the neurobiologist. Neuron 6:1-12.

Hays WL (1988) Statistics, pp 527-531. San Francisco: Holt, Rinehart and Winston.

Healy SD, Krebs JR (1992) Food storing and the hippocampus in corvids: amount and volume are correlated. Proc R Soc Lond [Biol] 248:241-245.

Jarrard LE (1993) Review: on the role of the hippocampus in learning and memory in the rat. Behav Neural Biol 60:9-26.

Krebs JR, Sherry DF, Healy SD, Perry VH, Vaccarino AL (1989) Hippocampal specialization of food-storing birds. Proc Natl Acad Sci USA 86:1388-1392.

Labbe R, Firl AC, Mufson EJ, Stein DG (1983) Fetal brain transplants: reduction of cognitive deficits in rats with frontal cortex lesions. Science 217:470-472.

Netto CA, Hodges H, Sinden JD, LePeillet E, Kershaw T, Sowinski P, Meldrum BS, Gray JA (1993) Effects of fetal hippocampal field grafts on ischemic-induced navigation in the water maze. Neuroscience 54:69-92.

Nieto-Sampedro M (1988) Growth factor induction and order of effects in CNA repair. In: Pharmacological approaches to the treatment of brain and spinal cord injury (Stein DG, Sabel BA, eds). New York: Plenum.

Nieto-Sampedro M, Wittemore SR, Needels DL, Larson J, Cotman CW (1984) The survival of brain transplants is enhanced by extracts from injured brain. Proc Natl Acad Sci USA 81:6250-6254.

Nishino H (1993) Intracerebral grafting of catecholamine producing cells and reconstruction of disturbed brain function. Neurosci Res 16:157-172.

O'Keefe J, Nadel L (1978) The hippocampus as a cognitive map. Oxford: Oxford UP.

Onifer SM, Low WC (1990) Spatial memory deficits resulting from ischemic-induced damage to the hippocampus is ameliorated by intrahippocampal transplants of fetal hippocampal neurons. Prog Brain Res 82:359-366.

Sherry DF, Vaccarino AL (1989) Hippocampus and memory for food caches in black-capped chickadees. Behav Neurosci 103:308-318.

Sherry DF, Jacobs LF, Gaulin SJ (1992) Spatial memory and adaptive specialization of the hippocampus. Trends Neurosci 15:298-303.

Sinden JD, Hodges H, Gray JA (1995) Neural transplantation and recovery of cognitive function. Behav Brain Sci 18:10-35.

Stein DG (1988) Practical and theoretical issues in the use of fetal brain tissue transplants to promote functional recovery from brain injury. In: Brain injury and recovery (Finger S, Levere TE, Almli CR, Stein DG, eds) New York: Plenum.

Stein DG, Glasier MM (1995) Some practical and theoretical issues concerning fetal brain tissue grafts as therapy for brain dysfunctions. Behav Brain Sci 18:36-45.

Stein DG, Mufson EJ (1987) Morphological and behavioural characteristics of embryonic brain tissue transplants in adult, brain damaged subjects. Ann NY Acad Sci 495:444-465.

Woodruff ML, Baisden RH, Nonneman AJ (1990) Transplantation of fetal hippocampus may prevent or produce behavioural recovery from hippocampal ablation and recovery persists after removal of the transplant. Prog Brain Res 82:367-376. 